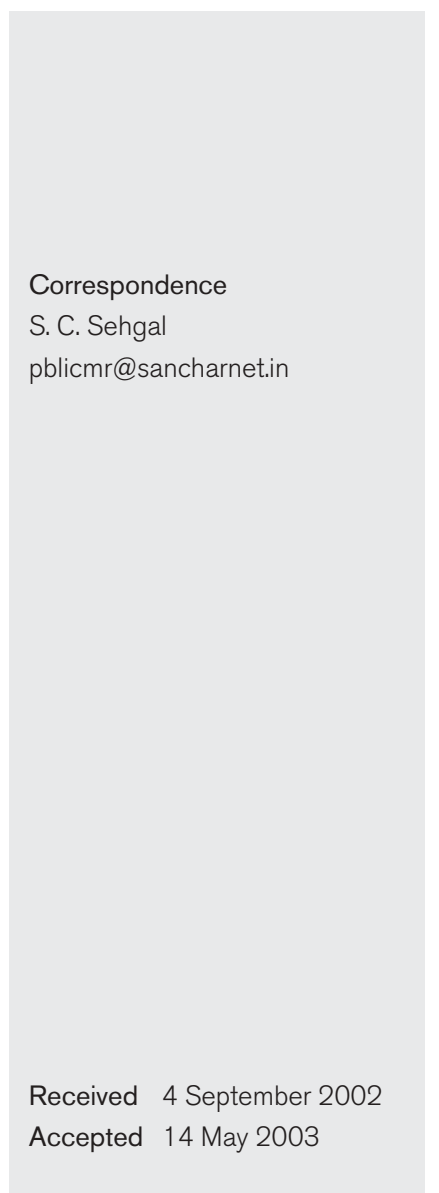

\title{
Field application of Lepto lateral flow for rapid diagnosis of leptospirosis
}

\author{
S. C. Sehgal, P. Vijayachari, A. P. Sugunan and T. Umapathi \\ National Leptospirosis Reference Centre, Regional Medical Research Centre (Indian Council of \\ Medical Research), Post Bag no. 13, Port Blair 744101, Andaman \& Nicobar Islands, India
}

\begin{abstract}
The Lepto lateral flow assay for leptospirosis was evaluated at a primary health centre in the Andaman Islands, where leptospirosis is endemic. One hundred and seventeen suspected patients were included in the study; acute serum samples were collected from all of them and convalescent samples from 104. The standard criteria for diagnosis of leptospirosis were: (i) isolation of leptospires from blood, (ii) seroconversion in microscopic agglutination test (MAT) with a minimum titre of 100 , (iii) a fourfold rise in titre in MAT or (iv) a MAT titre of 400 or more if only a single sample was available. The results of the lateral flow test were compared with these criteria. Lepto lateral flow had sensitivity of $52.9 \%$ (37/70) in the first week of illness and $86 \%(49 / 57)$ during weeks $2-4$. The corresponding specificities were respectively 93.6\% (44/47) and 89.4\% (42/47). The sensitivity was $34.3 \%(12 / 35)$ on days $2-3$ of the illness, $63.3 \%(14 / 22)$ on days $4-5$ and $84.6 \%(11 / 13)$ at the end of the first week. The test had a positive predictive value of $92.5 \%(37 / 40)$ during the first week and $90 \cdot 7 \%(49 / 54)$ subsequently. Corresponding negative predictive values were respectively $57 \cdot 1 \%(44 / 77)$ and $84 \%$ (42/50). Agreement of the results with the standard criteria was low during the first week, but high during weeks $2-4$, with a $\kappa$ value of $0 \cdot 7491$. The positivity rates of the tests showed a logarithmic relationship with the MAT titres of samples $\left(r^{2}=0.9271\right)$. All indices of validity and utility of lateral flow were similar to those of IgM ELISA and Lepto dipstick. The test can be performed at the bedside of the patient, as whole blood can also be used for testing.
\end{abstract}

\section{INTRODUCTION}

Leptospirosis is a zoonosis of worldwide distribution (WHO, 1999). Several outbreaks have been reported in recent years (Rathinam et al., 1997; Trevejo et al., 1998; WHO, 2000a, b). Clinical diagnosis of leptospirosis is difficult because of its protean manifestations. Because of the possibility of fatal complications like renal failure and pulmonary haemorrhage, laboratory confirmation of diagnosis is essential in order to take precautionary measures. Conventional laboratory methods include isolation of the organism and serological tests to demonstrate antibodies against leptospires. The culture technique is complicated, requires elaborate laboratory facilities and is time-consuming and the success rate is low. Culture does not fulfil the requirements of a physician, who needs rapid confirmation of clinical suspicion so as to take necessary precautions. Therefore, laboratory diagnosis usually depends upon serological techniques like microscopic agglutination test (MAT) and ELISA. MAT has several pitfalls. It requires a well-equipped laboratory, the maintenance of a large number of live Leptospira strains for use as antigens and technical expertise in reading and interpreting

Abbreviations: MAT, microscopic agglutination test; NPV, negative predictive value; $\mathrm{PHC}$, primary health centre; $\mathrm{PPV}$, positive predictive value. the results. Paired serum samples are needed for proper interpretation of the results. MAT titres are usually low during the acute stage of the disease and, hence, diagnosis based on a single serum sample is difficult (Faine, 1982). IgM ELISA is a useful screening technique for leptospirosis towards the end of the first week of illness (Terpstra et al., 1985). However, the limited shelf-life of reagents and the requirements for an ELISA reader to read the results and a continuous electrical supply to keep the test materials refrigerated limit its usefulness in developing countries.

The Lepto dipstick test, introduced recently, has overcome some of these problems, but it requires $3 \mathrm{~h}$ incubation before the results can be read (Gussenhoven et al., 1997; Sehgal et al., 1999). The Royal Tropical Institute (KIT), Amsterdam, The Netherlands, has recently developed another diagnostic test, namely Lepto lateral flow for rapid diagnosis of leptospirosis (Smits et al., 2001). It is based on the binding of specific IgM antibodies to the broadly reactive heat-extracted antigen prepared from the non-pathogenic Patoc 1 strain. IgM antibodies bound to the broadly reactive antigen are detected with an anti-human IgM gold conjugate contained within the test device. We evaluated this test under actual field conditions in an area highly endemic to leptospirosis. The 
parameters of evaluation were the validity of the test results, its utility and simplicity and its requirements in terms of skill and equipment.

\section{METHODS}

Patients and samples. A total of 117 patients attending the outpatient department of a Primary Health Centre (PHC) on South Andaman during the period October 1999-December 2000 were included in the study. All these patients had reported to the PHC within the first week of onset of illness and were suspected to have leptospirosis on clinical grounds. Blood samples were collected from all these patients on the day of reporting. A second sample was collected during weeks 2-4 of the illness. Second samples could be collected from only 104 patients. The 13 patients from whom second samples were not obtained were all diagnosed to have leptospirosis based on culture and/or MAT results on the first sample alone.

Isolation of leptospires. Blood culture was attempted on all first samples using EMJH semi-solid medium (Difco) following standard procedures (Faine, 1982).

Criteria for clinical suspicion of leptospirosis. The criteria for suspecting leptospirosis were the presence of fever, headache and body aches associated with any of the following symptoms: (i) calf muscle tenderness, (ii) bleeding tendencies, including subconjunctival haemorrhage, (iii) cough, haemoptysis and breathlessness, (iv) jaundice and (v) oliguria.

Lepto lateral flow. Lepto lateral flow kits were supplied by the KIT. The Lepto lateral flow test was performed according to the recommended procedure (Smits et al., 2001). Five microlitres undiluted serum or $10 \mu \mathrm{l}$ whole blood was added to the sample application well followed by $130 \mu \mathrm{l}$ sample fluid (diluent). After adding serum and sample solution, if only the control band became stained, the test was considered negative. If both test and control bands became stained, the test was considered positive. The results could be obtained within $15 \mathrm{~min}$. IgM ELISA was done following the standard procedure described by Terpstra et al. (1985). The Lepto lateral flow test was done on the first and second samples at the laboratory of the PHC itself. MAT, IgM ELISA and Lepto dipstick tests were performed at our laboratory.

Lepto dipstick and IgM ELISA. Lepto dipstick kits were supplied by the WHO South East Asia Regional Office (New Delhi, India) and the test was performed as reported earlier (Sehgal et al., 1999). IgM ELISA plates were prepared at our laboratory by coating the wells with heatextracted antigen of serogroup Icterohaemorrhagiae (serovar Copenhageni strain Wijnberg) using a standard procedure (Terpstra et al., 1985).

MAT. MAT was performed in microtitre plates using 12 live leptospiral strains, belonging to serogroups that are common in India, as antigens following the procedure described by Wolff (1954). The strains belonged to serogroups Grippotyphosa (serovar Grippotyphosa), Australis (serovar Australis), Ballum (serovar Ballum), Icterohaemorrhagiae (serovar Lai), Pyrogenes (serovar Pyrogenes), Tarassovi (serovar Tarassovi), Pomona (serovar Pomona), Autumnalis (serovar Rachmati), Canicola (serovar Canicola), Javanica (serovar Poi), Hebdomadis (serovar Hebdomadis) and Sejroe (serovar Hardjo). MAT was performed at dilutions of $1: 25,1: 50,1: 100,1: 200$ and $1: 400$. Those found positive at $1: 400$ were titrated up to end titres.

Criteria for laboratory confirmation. The criteria for confirmation of a diagnosis of current leptospiral infection were the same as those used in the evaluation of other rapid test systems (Sehgal et al., 1997).
Suspected patients fulfilling any of the following criteria were considered as a case of leptospirosis: (i) isolation of leptospires from blood, (ii) seroconversion in MAT from seronegative to a titre of at least 100, (iii) a fourfold rise in titre in MAT or (iv) a titre of 400 or more in MAT if only a single sample was available.

A titre of 400 was fixed for single samples because, at this cut-off titre, the specificity of MAT was found to be $87 \%$ during the first week in a high endemic area. Any higher cut-off titre would have negligible sensitivity (Vijayachari et al., 2001). If none of the above four criteria was fulfilled, the patient was considered as a control.

Measurements of test performance. Four indices, sensitivity and specificity (as measures of validity) and positive (PPV) and negative (NPV) predictive values (as measures of utility), were calculated for the Lepto lateral flow test, Lepto dipstick and IgM ELISA by comparing the results obtained in the respective tests with the standard criteria. These indices were calculated for two stages of the disease ( $\leqslant 7$ days and $8-30$ days). The $\kappa$ value for agreement between test results and standard diagnostic criteria, the standard error of $\kappa, \mathrm{Z}$ statistic and the corresponding $P$ values (Fleiss, 1981) were also calculated. Statistical calculations were done using the EPITABLE program of Epi Info version 6.03 (Dean et al., 1995). Percentage agreement between each pair of tests (lateral flow-dipstick, lateral flow-ELISA and dipstick-ELISA), $\kappa$ values and $\mathrm{Z}$ statistics were also calculated. Positivity rates of the lateral flow test on serum samples with different MAT titres were calculated to detect any association between antibody levels and test positivity rates. The lateral flow test was also done using whole blood in 28 cases to examine the agreement with test results obtained with serum samples. At the time of drawing blood from patients, $10 \mu \mathrm{l}$ was added from the syringe to the sample application well followed by $130 \mu \mathrm{l}$ sample fluid. Results were read after 15 min.

\section{RESULTS AND DISCUSSION}

Among the 117 study subjects, 70 fulfilled the standard criteria for diagnosis and were considered as cases of leptospirosis. Leptospires could be isolated in 28 patients. In the remaining 42 patients, the diagnosis was based on MAT results. Among the 28 culture-positive cases, 12 seroconverted from negative to titres greater than 100 and seven showed fourfold rises in titre. Only single serum samples were available from the remaining nine culturepositive patients. MAT was negative in five of them, two had MAT titres of 50 and one each had titres of 400 and 1600 . Among the 42 patients diagnosed on the basis of MAT results, 13 had fourfold rises in titre, 25 seroconverted and four had titres $\geqslant 400$.

Paired samples were available for all 47 patients in whom leptospirosis was ruled out, and none of them showed seroconversion, fourfold rises in titre or titres of more than 400. Because of the non-availability of second samples from 13 patients, the pre-test probability was different during week 1 and weeks 2-4: it was 59.8 \% (70/117) during week 1 and $54 \cdot 8 \%$ (57/104) during weeks $2-4$.

Sensitivity, specificity and predictive values of Lepto lateral flow, Lepto dipstick and IgM ELISA during week 1 and weeks $2-4$ of illness are summarized in Table 1 . The sensitivity of lateral flow increased from $53 \%$ in the first week to $86 \%$ during weeks $2-4$. Table 2 shows the positivity rates (sensitivity) of lateral flow at different durations of illness 
Table 1. Sensitivity, specificity, PPV and NPV of Lepto lateral flow, Lepto dipstick and IgM ELISA at different stages of illness

Sensitivity and specificity are given as percentages with no. positive/no. tested in parentheses. Thirteen patients were not available for testing during weeks 2-4; hence, the number of patients fell from 70 to 57 .

\begin{tabular}{|lccccccc|}
\hline $\begin{array}{l}\text { Duration of illness } \\
\text { (weeks) }\end{array}$ & Sensitivity & Specificity & PPV & NPV & $\boldsymbol{\kappa}$ value & Z value & $P$ (1-tailed) \\
\hline Lateral flow & & & & & & & \\
1 & $52 \cdot 9(37 / 70)$ & $93 \cdot 6(44 / 47)$ & $92 \cdot 5$ & $57 \cdot 1$ & $0 \cdot 4206$ & $5 \cdot 20$ & $0 \cdot 00000^{*}$ \\
$2-4$ & $86 \cdot 0(49 / 57)$ & $89 \cdot 4(42 / 47)$ & $90 \cdot 7$ & $84 \cdot 0$ & $0 \cdot 7491$ & $7 \cdot 65$ & $0 \cdot 00000$ \\
Lepto dipstick & & & & & & & \\
1 & $48 \cdot 6(34 / 70)$ & $85 \cdot 1(40 / 47)$ & $82 \cdot 9$ & $52 \cdot 6$ & $0 \cdot 3058$ & $3 \cdot 74$ & $0 \cdot 00009$ \\
$2-4$ & $87 \cdot 7(50 / 57)$ & $85 \cdot 1(40 / 47)$ & $87 \cdot 7$ & $85 \cdot 1$ & $0 \cdot 7282$ & $7 \cdot 43$ & $0 \cdot 00000$ \\
IgM ELISA & & & & & & & \\
1 & $50 \cdot 0(35 / 70)$ & $78 \cdot 7(37 / 47)$ & $77 \cdot 8$ & $51 \cdot 4$ & $0 \cdot 2642$ & $3 \cdot 13$ & $0 \cdot 00087$ \\
$2-4$ & $87 \cdot 7(50 / 57)$ & $87 \cdot 2(41 / 47)$ & $89 \cdot 3$ & $85 \cdot 4$ & $0 \cdot 7481$ & $7 \cdot 63$ & $0 \cdot 00000$ \\
\hline
\end{tabular}

${ }^{\star} 1$-tailed $P$ value less than $0 \cdot 00001$.

Table 2. Positivity of Lepto lateral flow, Lepto dipstick and IgM ELISA at different time intervals during week 1 of illness

\begin{tabular}{|lcccc|}
\hline $\begin{array}{l}\text { Duration of illness } \\
\text { (days) }\end{array}$ & Patients $(\boldsymbol{n})$ & \multicolumn{3}{c|}{ Positive tests $[\boldsymbol{n}(\%)]$} \\
\cline { 3 - 5 } & & $\begin{array}{c}\text { Lepto lateral } \\
\text { flow }\end{array}$ & Lepto dipstick & IgM ELISA \\
\hline $2-3$ & 35 & $12(34 \cdot 3)$ & $11(31 \cdot 4)$ & $11(31 \cdot 4)$ \\
$4-5$ & 22 & $14(63 \cdot 6)$ & $13(59 \cdot 0)$ & $13(59 \cdot 0)$ \\
$6-7$ & 13 & $11(84 \cdot 6)$ & $10(76 \cdot 9)$ & $11(84 \cdot 6)$ \\
\hline
\end{tabular}

during the first week: it was $34.3 \%$ on days $2-3,63.6 \%$ on days $4-5$ and $84.6 \%$ by the end of the first week. The increase in sensitivity during the second week was less marked; it was $84.6 \%$ at the beginning and $85 \%$ at the end of the week. The test detected antibodies in eight patients early in the first week of the illness when MAT was negative even at the initial dilution of $1: 25$. The specificity did not show large variations between week 1 and weeks $2-4$. It decreased from $93.6 \%$ in the first week to $89.4 \%$ during weeks $2-4$. The test was performed on 28 patients using both blood and serum. Sixteen of them were positive when tested with serum and 12 were negative. The results of the tests done using whole blood were identical, indicating that the test performs equally well with blood and serum. However, the bands were less visible after 1-2 h when the test was done using blood, as the whole strip became stained brown.

Lateral flow had PPVs above $90 \%$ during the first week and in weeks 2-4. However, it showed a decrease from week 1 to weeks 2-4. The lower pre-test probability and specificity might have contributed to this reduction in PPV during weeks 2-4. The NPV was low (57.1\%) during the first week, but increased to $84.0 \%$ during weeks $2-4$. Lepto dipstick and IgM ELISA also had similar but slightly lower predictive values (Table 1).

During the first week, the agreement between lateral flow results and the standard criteria for diagnosis was fair, as indicated by a $\kappa$ value greater than $0 \cdot 4$ (Fleiss, 1981). However, the agreement was poor for Lepto dipstick and IgM ELISA, with $\kappa$ values less than $0 \cdot 4$. During weeks $2-4$ of illness, lateral flow showed excellent agreement with the standard diagnostic criteria, with a $\kappa$ value close to $0 \cdot 75$. Lepto dipstick and IgM ELISA also had good agreement with standard diagnostic criteria during weeks $2-4$, but $\kappa$ values were lower than for lateral flow. The $\mathrm{Z}$ statistics for all the $\kappa$ values were large, with $P$ values less than 0.00001 in most cases, indicating that the observed agreement was much in excess of agreement by chance (Table 1). The three tests (lateral flow, dipstick and IgM ELISA) had good correlation. The percentage agreement, $\kappa$ values and $\mathrm{Z}$ statistics for each test combination are shown in Table 3. Agreement between lateral flow and dipstick and between dipstick and IgM ELISA was about $93 \%$. The agreement between lateral flow and IgM ELISA was slightly lower (86\%). 
Table 3. Agreement between Lepto lateral flow, Lepto dipstick and IgM ELISA

\begin{tabular}{|lcccc|}
\hline Comparison & Agreement (\%) & $\boldsymbol{\kappa}$ value & $\boldsymbol{Z}$ statistic & $\boldsymbol{P}$ value \\
\hline Lateral flow-dipstick & $92 \cdot 8$ & $0 \cdot 853$ & $12 \cdot 69$ & $0 \cdot 00000^{\star}$ \\
Lateral flow-IgM ELISA & $86 \cdot 0$ & $0 \cdot 716$ & $10 \cdot 66$ & $0 \cdot 00000$ \\
Dipstick-IgM ELISA & $93 \cdot 2$ & $0 \cdot 863$ & $12 \cdot 83$ & $0 \cdot 00000$ \\
\hline
\end{tabular}

${ }^{*} P$ value less than $0 \cdot 00001$.

Fig. 1 shows the trend in the positivity of the lateral flow test on serum samples with different MAT titres. The positivity rate increased from $21 \%$ in MAT-negative samples to $54.5 \%$ in samples with a titre of $1: 100$ and $81 \cdot 8 \%$ in samples with a titre of $1: 400$. The positivity rate was $100 \%$ in samples with a MAT titre of $1: 3200$. The positivity rates showed a good logarithmic agreement with the reciprocal titres $\left(r^{2}=\right.$ 0.9271).

IgM ELISA, Lepto dipstick and Lepto lateral flow showed similar results when compared with the standard criteria of diagnosis using culture and MAT. This is expected, since all these tests are based on the same principle. In weeks 2-4, all the tests showed good sensitivity. However, the sensitivity of all the tests was low in the first week.

Lepto lateral flow achieves the highest sensitivity during the third and fourth weeks of illness. It is this period when IgM antibody levels usually peak after infection (Ferrar, 1990). At this stage, all the indices (sensitivity, specificity, PPV, NPV) were around $90 \%$. Although the sensitivity was low when all the patients with duration of illness $\leqslant 7$ days were grouped together, the sensitivity showed an increasing trend when patients were subgrouped based on the duration of illness. When the duration of illness was 6-7 days, the sensitivity was very close to that for weeks $2-4(84 \cdot 6$ and $86 \%)$. Even when the duration of illness was only $4-5$ days, the test showed fairly good sensitivity (63.6\%). Lateral flow will be a valuable tool for the rapid diagnosis of leptospirosis when the duration of illness is more than 3 days.

Since the study subjects were suspected cases of leptospirosis attending a PHC, the pre-test probabilities were close to reallife situations. Hence, the predictive values give useful information about the utility of the test. The lateral flow test

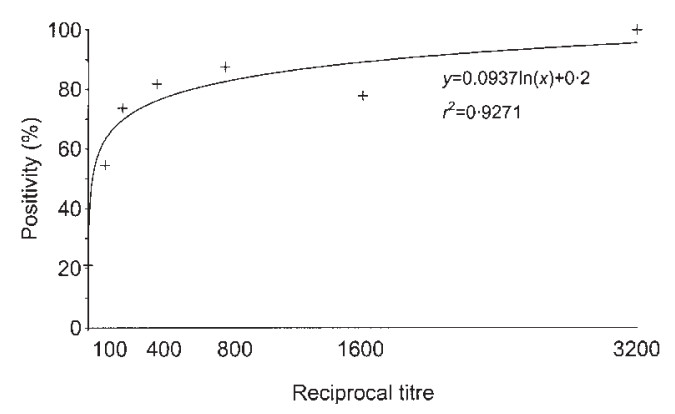

Fig. 1. Positivity rate in Lepto lateral flow at different MAT titres. has a PPV of $92.5 \%$, even during the first week. Therefore, a positive test almost confirms the diagnosis, though it might not be possible to rule out leptospirosis in the case of negative results during the first week because of the low NPV (57.1\%). The probability of having leptospirosis after a negative test (100-NPV \%) during the first week $(42.9 \%)$ is not substantially lower than the pre-test probability (59.8\%). Both Lepto dipstick and IgM ELISA also show this characteristic. As NPV is dependent on sensitivity, by day 5 or 6 of illness, when the sensitivity was above $80 \%$, the test might have an acceptable NPV. During weeks 2-4, the NPV of all the tests increased to about $85 \%$ and the difference between the pretest probability and post-test probability of a negative test became significant. All three tests detect anti-leptospiral IgM antibodies, and the agreement between them was good, with $\kappa$ values in the range $0 \cdot 713-0 \cdot 863$.

The possibility of getting a positive result in the test was strongly associated with the level of antibody in the sample, as shown by MAT titre. When the titre was $1: 100$, about $50 \%$ of the tests were positive and, at $1: 400$, more than $80 \%$ were positive. Beyond this, the increase in positivity rate was not as marked. Lateral flow specifically detects IgM antibodies, whereas MAT is responsive to both IgM and IgG (Faine, 1982). However, during the first month of illness, circulating IgM antibodies must play a major role in the agglutination of leptospires in MAT. This responsiveness to IgM by both the tests resulted in this observed association. Lateral flow is a qualitative test, and increase in the antibody levels beyond a particular stage does not significantly affect the chance of the test becoming positive.

The major advantage of Lepto lateral flow is its simplicity. It can be performed at the bedside of the patient, as whole blood can be used. We tested this technique on 28 samples using blood and serum. The results obtained with serum and blood were similar. The only difference was that the whole test strip becomes stained brownish after $1-2 \mathrm{~h}$ in the case of whole blood. Because of the possibility of doing the test directly with $10 \mu \mathrm{l}$ blood, even a drop of blood obtained by finger puncture might be sufficient. The test kit and sample solution do not require any special storage. During the study period, we stored the kits in the $\mathrm{PHC}$ at room temperature where the tests were conducted, and we did not observe any reduction in accuracy with time. In terms of validity of results and utility, lateral flow matches IgM ELISA and dipstick tests. However, Lepto lateral flow appears to be a more suitable test 
for hospitals and peripheral health care centres because of its simplicity and rapidity and the low requirements for skill, equipment and storage facilities for the kit and reagents.

\section{ACKNOWLEDGEMENTS}

The authors are grateful to Mr Henk L. Smits and Dr W. J. Terpstra, Department of Biomedical Research, Royal Tropical Institute (KIT), Amsterdam, The Netherlands, for providing Lepto lateral flow kits for evaluation, Dr C. K. Das and staff of the Primary Health Centre, Manglutan, South Andaman, for their help and co-operation and $\mathrm{Mr}$ Paritosh De, Laboratory Assistant, RMRC, Port Blair, for his assistance in the laboratory.

\section{REFERENCES}

Dean, A. G., Dean, J. A., Coulombier, D. \& 7 other authors (1995). Epi Info, version 6: a word processing, database, and statistics program for public health on IBM-compatible microcomputers. Centers for Disease Control and Prevention, Atlanta, GA, USA. http://www.cdc.gov/ epiinfo/ei6.htm

Faine, S. (1982). Guidelines for the Control of Leptospirosis. WHO offset publication no. 67, pp. 21-26. Geneva: World Health Organization.

Ferrar, W. E. (1990). Leptospira species (leptospirosis). In Principles and Practice of Infectious Diseases, pp. 1813-1827. Edited by G. L. Mandel, R. G. Douglas \& J. E. Bennett. New York: Churchill Livingstone.

Fleiss, J. L. (1981). The measurement of interrate agreement. In Statistical Methods for Rates and Proportions, 2nd edn, pp. 217-225. New York: Wiley.

Gussenhoven, G. C., van der Hoorn, M. A. W. G., Goris, M. G. A., Terpstra, W. J., Hartskeerl, R. A., Mol, B. W., van Ingen, C. W. \& Smits, H. L. (1997). LEPTO dipstick, a dipstick assay for detection of Leptospiraspecific immunoglobulin $\mathrm{M}$ antibodies in human sera. J Clin Microbiol $35,92-97$.
Rathinam, S. R., Rathnam, S., Selvaraj, S., Dean, D., Nozik, R. A. \& Namperumalsamy, P. (1997). Uveitis associated with an epidemic outbreak of leptospirosis. Am J Ophthalmol 124, 71-79.

Sehgal, S. C., Vijayachari, P. \& Subramaniam, V. (1997). Evaluation of Leptospira microcapsule agglutination test (MCAT) for serodiagnosis of leptospirosis. Indian J Med Res 106, 504-507.

Sehgal, S. C., Vijayachari, P., Sharma, S. \& Sugunan, A. P. (1999). LEPTO dipstick: a rapid and simple method for serodiagnosis of acute leptospirosis. Trans R Soc Trop Med Hyg 93, 161-164.

Smits, H. L., Eapen, C. K., Sugathan, S. \& 8 other authors (2001). Lateral-flow assay for rapid serodiagnosis of human leptospirosis. Clin Diagn Lab Immunol 8, 166-169.

Terpstra, W. J., Ligthart, G. S. \& Schoone, G. J. (1985). ELISA for the detection of specific IgM and IgG in human leptospirosis. J Gen Microbiol 131, 377-385.

Trevejo, R. T., Rigau-Perez, J. G., Ashford, D. A. \& 14 other authors (1998). Epidemic leptospirosis associated with pulmonary hemorrhage - Nicaragua, 1995. J Infect Dis 178, 1457-1463.

Vijayachari, P., Sugunan, A. P. \& Sehgal, S. C. (2001). Evaluation of microscopic agglutination test as a diagnostic tool during acute stage of leptospirosis in high and low endemic areas. Indian J Med Res 114, 99-106.

WHO (1999). Leptospirosis, Australia, January 1998-March 1999. WHO/FAO Collaborating Centre for Reference and Research on Leptospirosis. Wkly Epidemiol Rec 74, 113-118.

WHO (2000a). Leptospirosis, India. Report of the investigation of a post-cyclone outbreak in Orissa, November 1999. Wkly Epidemiol Rec $75,217-223$.

WHO (2000b). Outbreak news. Wkly Epidemiol Rec 75, 313.

Wolff, J. W. (1954). In The Laboratory Diagnosis of Leptospirosis, pp. 39-62. Springfield, IL: Charles C. Thomas. 